[W] H. Wolter, On the problem of the last root for exponential terms, Preprint 58 (1983) der Sektion Mathematik der Humboldt-Universität zu Berlin, to appear in Z. Math. Logik Grundlag. Math.

[Wi] A. Wilkie, On exponential fields, Preprint.

SEKTION MATHEMATIK DER HUMBOLDT-UNIVERSITÄT,

DDR-1086, Berlin PSF 1297, GDR

\section{Uniform quotients of metrizable spaces}

\section{J. Vilímovský (Praha)}

Abstract. The easiest possible example of a metrizable uniform space having a nonmetrizable uniform quotient is given. Using this example all metrizable spaces having metrizable uniform quotients only are fully described.

In the literature several sufficient conditions for a uniform quotient of a metric space to be metrizable are treated e.g. [1], [2], [4]. The first attempt to bring a concrete example of a nonmetrizable uniform quotient of a metric space appeared in [4], and two much simpler examples appeared later in [3]

In the sequel $f: X \rightarrow Y$ is a uniformly continuous onto mapping between uniform spaces. $f$ is called a uniform quotient mapping if $Y$ is endowed with the finest uniformity making $f$ uniformly continuous. Himmelberg [2] strengthens the latter concept defining so called uniformly pseudoopen mappings (i.e. the images of uniform vicinities of the diagonal are uniform vicinities) and proves that a uniformly pseudoopen image of a metrizable space is metrizable. We start with another strengthening of uniform quotient mappings which seems to be more convenient (see Remark 1) for our problem.

DefinItron 1. Let $f: X \rightarrow Y$ be a uniformly continuous mapping from $X$ onto $Y$. $f$ will be called uniformly conservative if for every uniform cover $\mathscr{U}$ of $X$ the cover

$$
\tilde{f}(\mathscr{U})=\left\{f\left[\operatorname{St}\left(f^{-1}(y), \mathscr{U}\right)\right] ; y \in Y\right\}
$$

is uniform on $Y$.

It might be easily verified that every uniformly pseudoopen mapping is uniformly conservative and every uniformly conservative mapping is a uniform quotient.

Proposirion 1. If $f: X \rightarrow Y$ is uniformly conservative (onto), $X$ metrizable, then $Y$ is metrizable as well.

Proof. Take an arbitrary uniform cover $\mathscr{V}$ of $Y$, choose a uniform star-refinement $\mathscr{W}$ of $\mathscr{V}$ and set $\mathscr{U}=f^{-1}(\mathscr{W})$. Then for every $y \in Y$ we have

$$
f\left[\operatorname{St}\left(f^{-1}(y), \mathscr{u}\right)\right] \subset \operatorname{St}(y, \mathscr{W})
$$


Therefore every uniform cover of $Y$ may be refined by a cover of the form $f(\mathcal{U})$ Therefore every uniform cover $X . f$ is uniformly conservative, $X$ has a countable basis for uniform covers, so $Y$ has a countable basis for covers as well.

Now we present two results which will show that under some conditions quotients are uniformly conservative.

Proposition 2. Suppose $f: X \rightarrow Y$ is continuous and onto, $X$ compact, (hence $f$ is a uniform quotient), then $f$ is uniformly conservative.

Proof (standart). Take $y \in Y$ and an open neighborhood $U$ of $f^{-1}(y)$. For every $z$ distinct from $y$ take $V(z), W(z)$ disjoint open in $Y$ such that $z \in V(z), y \in W(z)$. The cover

$$
\{U\} \cup\left\{f^{-1}[V(z)] ; z \in X \backslash\{y\}\right\}
$$

is an open cover of $X$, we may choose a finite subcover

$$
\{U\} \cup\left\{f^{-1}\left[V\left(z_{i}\right)\right] ; i=1, \ldots, k\right\} .
$$

The set $W=\bigcap_{i=1}^{k} W\left(z_{i}\right)$ is an open neighborhood of $y$.

If $x \in W$ then $f^{-1}(x) \cap f^{-1}\left[V\left(z_{i}\right)\right]=\varnothing$ for all $i$; hence $f^{-1}(x) \subset U$, and hence $W \subset f[U]$, therefore $f[U]$ is a neighborhood of $y$. Take any open cover $\mathscr{U}$ of $X$, the set $f\left[\operatorname{St}\left(f^{-1}(y), \mathscr{U}\right)\right]$ is a neighborhood of $y$ for all $y \in Y$, so $f(\mathscr{U})$ is a uniform cover of $Y$.

COROLLARY 1. Every uniform quotient of a compact metrizable space is metrizable.

Remark 1. Proposition 2 remains no more true if we write uniformly pseudoopen instead of uniformly conservative as shows the following easy example:

Put $X=[0,3], Y=[0,2]$ compact intervals, $f(x)=x$ for $x \in[0,1]$, $f(x)=x-1$ for $x \in[2,3], f(x)=1$ otherwise. $\tilde{f}$ is obviously uniformly conservative but not uniformly pseudoopen.

Propositron 3. Let $f: X \rightarrow Y$ be a uniform quotient,

$$
Y_{1}=\left\{y \in Y ;\left|f^{-1}(y)\right| \geqslant 2\right\} .
$$

If the family $\left\{f^{-1}(y) ; y \in Y_{1}\right\}$ is uniformly discrete in $X$ then $f$ is uniformly conservative.

Proof. Take a uniform cover $\mathcal{U}$ of $X$. We may suppose that for every $y, z \in Y_{1}$ the sets $\operatorname{St}\left(f^{-1}(y), \mathscr{U}\right)$ and $\operatorname{St}\left(f^{-1}(z), \mathscr{U}\right)$ are disjoint. Suppose $\mathscr{V}$ is a uniform star-refinement of $\mathscr{U}, z \in Y$.

a) If $z \in Y_{1}$, then easily $\operatorname{St}(z, f(\mathscr{V})) \subset f\left[\operatorname{St}\left(f^{-1}(z), \mathscr{U}\right)\right]$

b) If $z \notin Y_{1}$ and $\operatorname{St}\left(f^{-1}(z), \mathscr{V}\right)$ does not intersect any $f^{-1}(y)$ for $y \in Y_{1}$, then evidently $\operatorname{St}(z, \tilde{f}(\mathscr{V})) \subset f\left[\operatorname{St}\left(f^{-1}(z), \mathscr{U}\right)\right]$.

c) If $z \notin Y_{1}$ and $\operatorname{St}\left(f^{-1}(z), \mathscr{V}\right)$ intersects some $f^{-1}(y)$ with $y \in Y_{1}$, then this $y$ is unique and we may find $U \in \mathscr{U}$ containing $\operatorname{St}\left(f^{-1}(z), \mathscr{V}\right)$ and intersecting $f^{-1}(y)$, hence $\operatorname{St}(z, \tilde{f}(\mathscr{V})) \subset f\left[\operatorname{St}\left(f^{-1}(y), \mathscr{U}\right)\right]$.

So $\tilde{f}(\mathscr{U})$ is star-refined by $f(\mathscr{V})$, hence $f$ is uniformly conservative.
COROLlaRY 2. If $X$ is mctrizable, $f$ is a quotient mapping such that the family of all nontrivial fibres is uniformly discrete, then the quotient ispace is metrizable.

Proposirion 4. Suppose $X$ is metrizable and every quotientiof it is also metrizable, $Y$ is a subspace of $X$. Then every quotient of $Y$ is metrizable $:$

Proof. Suppose $f: Y \rightarrow Q$ is uniform quotient, let us embed $\dot{Q}$ as a uniform subspace into some injective space $Z$ and extend $f$ to a uniformly continuous $\bar{f}: X \rightarrow Z$. If we take the quotient uniformity on $f[Z]$, then this uniform space has to be metrizable and contains $Q$ as a uniform subspace. So $Q$ is metrizable as well.

Corollary 3. Livery precompact metrizable space has metrizable quotients only.

Let us turn our attention to nonmetrizable quotients now. Let us denote by $D_{2}$ the adjacent sequence (see [6], where also the strange notation is justified), that is the set $N \times 2$ with the uniformity metrized by the metric $d(x, x)=0, d(\langle n, 0\rangle,\langle n, 1\rangle)$ $=1 / n, d(x, y)=1$ otherwise. It is proved in [5] that every metrizable space which is not uniformly discrete contains as a uniform subspace either a cauchy sequence or a copy of $D_{2}$. Every quotient of a cauchy sequence must be metrizable due to its precompactness, so if we prove (and we shall prove it) that $D_{2}$ has a nonmetrizable quotient, it will be the easiest possible example.

EXAMPLE 1. Take $f: D_{2} \rightarrow \omega$ defined as $f(1,1)=0, f(n, 0)=f(n+1,1)=n$. Then the corresponding quotient uniformity $Q$ on $\omega$ is not metrizable.

Proof. We start with the following notation: if $f: X \rightarrow Y$ is a uniform quotient and if $X$ is metrized by a metric $d$, we shall denote by $d_{f}$ the following pseudometric on $Y$ :

$$
d_{f}(x, y)=\inf \sum_{0}^{n-1} d\left(f^{-1}\left(x_{i}\right), f^{-1}\left(x_{i+1}\right)\right)
$$

where $x_{0}=x, x_{n}=y$ and the infimum is taken over all such chains. Obviously $f$ is uniformly continuous into the pseudometric space $\left(Y, d_{f}\right)$.

The following observation is due to Marxen:

LEMMA ([4]). Suppose $Y$ is metrized by $\sigma$, then there is a metric $d^{\prime}$ uniformly, equivalent to $d$ such that $d_{s}^{\prime}$ is uniformly equivalent to $\sigma$.

Using this idea we come back to our example. Suppose $Q$ is metrizable, we have some $\sigma$ metrizing $D_{2}$ such that $\sigma_{f}$ metrizes $Q . \sigma$ may be characterized by some sequence $\left\{a_{n}\right\}$ of real numbers such that $\left.1 \geqslant a_{n}\right\rangle 0, \sigma(\langle n, 0\rangle,\langle n, 1\rangle)=a_{n}$. We may and shall suppose ( $Q$ has a quotient uniformity) that $\sum a_{n}=\infty$. Now define a new metric $\sigma^{1}(\langle n, 0\rangle,\langle n, 1\rangle)=b_{n}$ on $D_{2}$ (equivalent to $\sigma$ ) as follows:

For every $k \in N$ denote $n(1)=1$, otherwise $n(k)$ is the first natural number such that

(i) $a_{n(k)}+\ldots+a_{n(k)+k-1}<\frac{1}{k}$,

(ii) $a_{n(k)} \geqslant a_{n(k-1)+k-1}$. 
For $n(k) \leqslant n \leqslant n(k+1)$ we define $b_{n}=\frac{1}{k}$.

For all natural $n$ we have $b_{n} \geqslant a_{n}$; hence $\sigma^{1} \geqslant \sigma$, hence $\sigma_{f}^{1} \geqslant \sigma_{f}$.

On the other hand, for any $k \geqslant 2$ there are points $v=n(k)-1, w=n(k)+k-1$ in $Q$ such that

$$
\begin{aligned}
& \sigma_{f}(v, w)=\sum_{i=1}^{k-1} a_{n(k)+i}<\frac{1}{k} \\
& \sigma_{f}^{1}\left(v, w^{\prime}\right)=\sum_{n=n(k)}^{n(k)+k-1} b_{n}=1
\end{aligned}
$$

Therefore $\sigma_{f}^{1}$ is not uniformly equivalent to $\sigma_{f}$, and hence $\sigma_{f}$ does not metrize $Q$, which contradicts the assumption.

Remark 2. Recall that the uniform weight of a space is the smallest cardinality of a base of uniform covers. Hušek and Pelant [3] proved that if $Y$ is a uniform quotient of a metrizable space, then its uniform weight is less or equal to $\operatorname{cof}\left({ }^{\circ} \omega\right)$. (The latter symbol stands for the smallest cardinality of a cofinal set in the set of all mappings on $\omega$ into $\omega$ with the pointwise order.) If one looks carefully how the uniform covers in the space $Q$ from Example 1 look like, one can easily see that the uniform weight of $Q$ is just $\operatorname{cof}\left({ }^{(} \omega\right)$, so our example is as "far from being metrizable" as possible.

Now we are prepared to prove the main result:

THEOREM. The following properties of a metrizable uniform space $X$ are equivalent:

(1) Every uniform quotient of $X$ is metrizable,

(2) $X$ does not contain $D_{2}$ as a uniform subspace,

(3) Every uniform subspace of $X$ is uniformly discrete or contains a Cauchy sequence as a uniform subspace.

(4) The completion $\hat{X}$ of $X$ is of the form $L \cup(X \backslash L)$, where $L$ is compact and for every $\varepsilon>0$ the subspace $\widehat{X} \backslash \mathcal{O}_{\varepsilon}(L)$ is uniformly discrete $\mathcal{O}_{\varepsilon}(L)$ stands for the $\varepsilon$-neighborhood of $L$ ).

Proof. (1) $\Rightarrow(2)$ follows from Proposition 4 and Example 1 .

$(2) \Leftrightarrow(3)$ is contained in the remark foregoing Example 1 .

$(3) \Rightarrow(4)$. It might be easily observed that the condition (2) is closed under completions, so if $X$ fulfils (3), so does $\hat{X}$. Take all copies of a cauchy sequence in $\bar{X}$ and denote by $L$ the set of all their limit points. (3) implies that $L$ is compact. If $X \backslash \mathcal{O}_{S}(L)$ is not uniformly discrete for some $\varepsilon>0$, it must contain a cauchy sequence not converging to a point in $L$, which contradicts the definition of $L$.

$(4) \Rightarrow(1)$. Using Proposition 4 we may suppose that $X$ is complete. Let $\varrho_{1}$ be any metric metrizing the uniformity of $X$. If $f: X \rightarrow Y$ is a quotient mapping, using Proposition 2 we may find a metric $\sigma$ metrizing $L$ such that the corresponding $\sigma_{S \mid L}$ metrizes the quotient uniformity on $f[L]$, the latter being a subspace of $Y$. Take $\varrho_{2}$ any uniformly continuous extension of $\sigma$ over all of $X$. Set $\varrho=\max \left(\varrho_{1}, \varrho_{2}\right)$. $\varrho$ is generating the uniformity of $X$. For every $x \in X$ take

Now define for $x, y \in X$ :

$$
L_{x}=\{y \in L ; \varrho(x, y)=\varrho(x, L)\} \neq \varnothing .
$$

$$
\begin{aligned}
& d(x, x)=0, \\
& d(x, y)=\inf _{\substack{u \in L_{x} \\
v \in L_{y}}}(\varrho(x, u)+\varrho(u, v)+\varrho(v, y)) \text { if } x \neq y .
\end{aligned}
$$

It is easy to see that $d$ is a metric on $X, d \geqslant \varrho$ and $d(x, y)=\varrho(x, y)$ for $x, y$ in $L$. For every $\varepsilon>0$ there is $\eta(\varepsilon)>0$ such that $\varrho(x, y) \geqslant \eta(\varepsilon)$ for $x, y \notin \mathcal{O}_{\varepsilon}^{e}(L)$. Put $\delta=\min \left(\frac{\varepsilon}{5}, \eta\left(\frac{\varepsilon}{5}\right)\right)$. Then for $\varrho(x, y)<\delta$ we have $\varrho(x, L)<\frac{\varepsilon}{5}, \varrho(y, L)<\frac{\varepsilon}{5}$.

For arbitrary $\mu>0$ we may find $u \in L_{x}, v \in L_{y}$ so that

$$
\begin{aligned}
& d(x, y)<\varrho(x, u)+\varrho(u, v)+\varrho(v, y)+\mu, \\
& \varrho\left(u, v<\varrho(u, x)+\varrho(x, y)+\varrho(v, y)<\frac{3}{5} \varepsilon .\right.
\end{aligned}
$$

So we have $d(x, y) \leqslant \varepsilon$, and hence $d$ is uniformly equivalent to $\varrho$. Now it is easy to check that the corresponding metric $d_{f}$ metrizes the quotient uniformity on $Y$.

\section{References}

[1] E. Cech, Topological spaces (revised edition by Z. Frolík, M. Katětov), Academia, Prague 1966

[2] C. J. Himmelberg, Quotient uniformities and uniformly pseudoopen mops, Bull Inst Acad. Sinica 2 (1974), 357-369.

[3] M. Hušek, J. Pelant, Uniform weight of uniform quotients, Comment. Math. Univ. Carolinae 24 (1983), 335-340.

14] D. Marxen, Uniform quotients of metric spaces, Fund. Math. 108. (1980), 67-75.

[5] G. Tashjian, J. Vilimovský, Coreflectors not preserving the interval and Baire partitions of uniform spaces, Proc. Amer. Math. Soc. 77 (1979), 257-263.

[6] J. Vilimovský, Several extremal coreflective classes in uniform spaces, Czechoslovak Math. J. 30 (1980), 569-578.

INSTITUTE OF MATHEMATICS

CZECHOSLOVAK ACADEMY OF SCIENCES

Zitni 25

115 67 Prague
Crechoslovakia

Received 23 May 1985 